\title{
6 Schluss
}

Die Bücher des Johann Jacob Wecker waren äusserst beliebt, und das über eine lange Zeit hinaus. Davon zeugen über hundert Ausgaben, Übersetzungen ins Deutsche, Französische und Englische, die Tatsache, dass auch Mitte des 18. Jahrhunderts noch Publikationen im Namen Weckers veröffentlicht wurden sowie jene, dass einige von ihnen noch heute problemlos über ebay.com erstanden werden können. ${ }^{917}$ Die Weckersche Buchproduktion war nicht nur umfang-, sondern auch variantenreich, wie die vorliegende Studie gezeigt hat. Sie umfasste medizinische, aber auch philosophische Texte unterschiedlicher Formen und Gattungen, wobei die Bücher, die aus der Rückschau als Books of Secrets bezeichnet werden, am populärsten waren. ${ }^{918}$ Sie enthalten Wissen aus unterschiedlichsten Traditionen, Zeiten und Räumen. Diese Art der Textproduktion war nur möglich dank der Praktik der Kompilation, die dieser zu Grunde lag: Sie war nicht nur verantwortlich für die Vielfalt der Inhalte, sondern auch für die grosse Anzahl an Publikationen, die unter dem Namen Weckers überliefert ist.

Hinter der Praktik der Kompilation stand, so die These, nicht ein Autor oder eine Autorin allein, sondern standen Kompilationsnetzwerke, die eine rasche Textproduktion ermöglichten und für die Vielfalt und Fülle von und innerhalb von Kompilationen verantwortlich waren. Deren Textbestandteile, die gesammelt, geordnet und neu publiziert wurden, entstammten Büchern, weshalb die Kompilation eine Textproduktionspraktik ist, bei der Bücher aus Büchern hergestellt werden. Dies durch die Ausführung sogenannter Buchpraktiken - also auf die Produktion von Büchern bezogene Praktiken -, welche die Grundlage der frühneuzeitlichen Kompilationsproduktion bildeten. Diese Praktiken sind anhand der Weckerschen Textproduktion besonders gut greifbar, da sie sowohl in der Korrespondenz als auch in den Büchern selbst immer wieder hervortreten. So sind beispielsweise Änderungen und Kürzungen direkt im Text erkennbar. Den Textelementen kann auch deshalb gut nachgegangen werden, weil Wecker und seine Kollaborierenden die Autoritäten, manchmal gar die Bücher, denen sie Wissen entnommen hatten, offenlegten. Bei dieser Art der Textproduktion ging es folglich darum, aus Büchern Bücher zu machen und diese - wie auch das darin gespeicherte Wissen - rasch zu vermehren und verbreiten.

Dies gilt auch für die Weckerschen Books of Secrets. Damit argumentiere ich gegen die von William Eamon angeführte These, die besagt, dass sich Books of

917 Siehe z.B. Ebay. Online unter: https://www.ebay.com/itm/Wecker-Occult-De-secretis-libriXVII-60-Engravings-1588-/323653148156 [24.02.2021].

$918 \mathrm{Zu}$ den Weckerschen Publikationen siehe Kapitel 1 sowie die Liste derselben im Anhang, 7.4.

Ә OpenAccess. ( 2022 Simone Zweifel, publiziert von De Gruyter. (cc)BY Dieses Werk ist lizenziert unter einer Creative Commons Namensnennung 4.0 International Lizenz. 
Secrets dadurch auszeichnen, dass deren Rezepte durch ein experimentelles, „Vor-Baconsches“ Verfahren vor dem Druck getestet worden seien. Er schreibt: „[...], the professors of secrets brought into sharp relief the contrasting authorites of experience versus book learning, and came down firmly on the side of the former. “919 Das vorliegende Buch geht jedoch davon aus, dass Bücher - und nicht Experimente - die Grundlage der Secreti-Produktion bildeten. Dies kann nicht nur für die Weckerschen Publikationen angenommen werden, sondern auch für frühere Books of Secrets, die sich selbst als kompilatorisch beschreiben. ${ }^{920}$ Die in diesen Büchern verwendete Praktik der Wissensproduktion war demnach die der Kompilation - und nicht eine etwaige Praktik des Testens oder eine andere Form der Wissensproduktion. Dies ist deshalb von Bedeutung, weil nur durch Kompilation derart rasch derart viel Wissen gesammelt und verbreitet werden konnte: ein Testen aller Rezepte hätte dies unmöglich gemacht. Die Kompilation war denn auch der ausschlaggebende Faktor für die Multiplizierung der Wissensbestände in kurzer Zeit.

Doch wie konnte derart rasch viel und unterschiedliches Buchwissen akkumuliert und veröffentlicht werden? Diese Studie hat gezeigt, dass dies nur möglich war dank der Kompilationsnetzwerke, die hinter der Produktion von Kompilationen standen. Deren Akteurinnen und Akteure informierten sich gegenseitig über mögliche Wissenssammlungen, aus denen kompiliert werden konnte, sie unterstützten sich bei der Beschaffung von Büchern, sie tauschten sich über inhaltliche Details aus, sie korrespondierten und überbrachten Briefe, Bücher und Geschenke als Dank für etwaige Korrekturen sowie geleistete Arbeiten. Die Konzeptualisierung des Kompilationsnetzwerks erfolgte im Kapitel 3 durch eine von den Quellen ausgehenden mikrohistorischen Herangehensweise, die in einem spiralförmigen Verfahren immer wieder mit anderen Ideen aus der Forschung, insbesondere aus der Buchforschung und der Praxistheorie, in Bezug gesetzt wurde. Grundlage waren - für das Konzept wie auch für die gesamte vorliegende Arbeit - die Publikationen und Briefe Johann Jacob Weckers sowie seiner Nachfolgerinnen und Nachfolger. Sie ermöglichten einen neuen Einblick in die Praktik der zeitgenössischen Kompilationsproduktion. Durch den Miteinbezug der Briefe geht die Studie über die meisten Forschungen zu Books of Secrets hinaus, die sich auf die Erforschung der Bücher beschränken. ${ }^{921}$ Dank der Korrespondenz wurden Akteurinnen und Akteure der Produktion von Books of Secrets sichtbar, die bis-

919 Eamon: Science and the Secrets of Nature, 1996, S. 161.

920 So argumentiert etwa das Piemontese: Secreti del Reverendo Donno Alessio Piemontese, 1555.

921 Siehe z. B. Eamon: Science and the Secrets of Nature, 1996; Kavey: Books of Secrets, 2007. 
lang aussen vor gelassen wurden, wie etwa Botinnen und Boten, die eine wichtige Rolle innehatten: Sie mussten ein Buch abliefern, damit daraus kompiliert werden konnte. Dies war nicht immer der Fall, wie es folgendes Zitat verdeutlicht: „Mein bott ist jetz bei 8 wůchen auss, weiss auch nitt wie ess stodt, ob er tod oder lebendig [...].“922 Ging ein Bote „verloren“, so kam auch der Brief oder das Buch nicht an, was sich auf den am Ende gedruckten Text auswirken konnte. Aus diesem Grund sind auch Botinnen und Boten als Teil des Kompilationsnetzwerks zu berücksichtigen.

Das Kompilationsnetzwerk wurde in diesem Buch als Geflecht unterschiedlicher Akteurinnen und Akteuren sowie Elementen, die relational in Zusammenarbeit eine Kompilation produzierten, konzeptualisiert. Teil eines Kompilationsnetzwerks waren Menschen unterschiedlicher sozialer Schichten in verschiedenen Positionen. Dazu zählen unter anderem Personen, die Text herstellten, Menschen, die dieses ermöglichten, Botinnen und Boten, Personen, die in der Druckerei tätig waren sowie weitere, deren Arbeit sich direkt auf die Kompilationsproduktion auswirkte. Zentrale Akteure vieler Weckerscher Kompilationsnetzwerke waren Theodor Zwinger und Pietro Perna. Während Perna für den Druck verantwortlich war, übernahm Zwinger unterschiedlichste Aufgaben: Er war unter anderem Ideengeber für neue Bücher, aus denen kompiliert werden konnte, er korrigierte und kommentierte, er vermittelte bei Druckern und schrieb Vorreden für Wecker. Doch nicht nur Zwinger und Perna waren Teil dieses Netzwerks, sondern etwa auch Anna Wecker, die Frau Johann Jacob Weckers, die Briefe überbrachte und später an Neueditionen Weckers beteiligt war, als dieser bereits verstorben war. Ebenfalls beteiligt war Samuel Grynæus, der für Wecker Bücher aus Frankfurt, der Stadt der Buchmesse, beschaffte. Involviert waren jedoch nicht nur Menschen, sondern auch Dinge. Besonders Bücher konnten einen grossen Einfluss auf den am Ende produzierten Text haben, allein schon durch ihr Vorhandensein oder Nichtvorhandensein. Daneben waren in der Frühen Neuzeit auch Briefe in ihrer kommunikativen und sozialen Funktion unabdingbar für das Zustandekommen von Kompilationen.

Teil eines Kompilationsnetzwerks waren demnach Personen sowie Dinge, die den am Ende produzierten Text beeinflussten. Dies gilt auch für äussere Faktoren, die ebenfalls auf den Text einwirken konnten, wie etwa die Frankfurter Buchmesse: Der Zeitpunkt derselben konnte beispielsweise dazu führen, dass ein Buch - nicht wie ursprünglich geplant - ohne Bilder erschien. ${ }^{923}$ Dies konnte den Gesamttext verändern. Solche äusseren Faktoren wurden als mitbeeinflussende

922 Wecker, Johann Jacob: Brief an Theodor Zwinger. UB Basel, Frey-Gryn Mscr II 5:Nr. 94. 923 Siehe dazu Kapitel 3.2 zur Praxistheorie. 
Elemente ins Konzept des Kompilationsnetzwerks, das ein relationales ist, integriert. Relational deshalb, weil unterschiedliche Elemente und Personen in diversen Konstellationen auf verschiedene Kompilationen Einfluss nahmen, wobei einzelne Akteurinnen oder Akteure bei einem Buch stärker involviert sein konnten als bei einem anderen. Bei der Weckerschen Buchproduktion wurde etwa Theodor Zwinger mal mehr, mal weniger miteinbezogen. Für die De Secretis XVII von 1582 war er beispielsweise besonders wichtig, da er zwischen Wecker und Pietro Perna vermittelte, als sich die beiden nicht einig über die Publikation dieses Buches waren. Ohne diese Vermittlung wäre diese Ausgabe, die als die erfolgreichste Weckersche Publikation betrachtet werden kann, wohl nicht erschienen.

Die Personen der Kompilationsnetzwerke übten Buchpraktiken aus, die zur Herstellung von Büchern führten. $\mathrm{Zu}$ den Buchpraktiken gehörte das Sammeln von Wissen ebenso das Schreiben: „Dan über daß, daß ich grosen costen an büchern, [...] so hab ich darmehr mitt schreiben, colligieren, vil arbeit gehabt, vnd vil an meiner prattic versaůmpt. “924 In diesem Brief beklagte sich Wecker, dass er viel Zeit seiner medizinische Praxis für das Schreiben und Colligieren - also das Sammeln von Wissen - versäumt habe. Zu den Buchpraktiken von Kompilationen zählten jedoch nicht nur diese beiden, sondern auch die Beschaffung von Büchern, denen Text- oder auch Bildbestandteile entnommen werden können, das Korrespondieren, das Lesen, das Übersetzen, das Kompilieren und Ordnen von Wissen, das Verfassen von Widmungen, das Überarbeiten, das Korrigieren und das Auskoppeln von Textteilen zur Herstellung neuer Bücher. Auch das Aufbauen und das Aufrechterhalten sozialer Beziehungen konnten und können Buchpraktiken sein, wenn durch diese Verbindungen Drucke erst möglich wurden oder werden. Diesen Praktiken wurde in Kapitel 4 nachgegangen. Das Kapitel zeigte auf, wie unterschiedlich die einzelnen Schritte bis zur Publikation waren und dass an den meisten mehrere Akteurinnen, Akteure und Elemente beteiligt waren, was eine wichtige Grundlage des Konzepts des Kompilationsnetzwerks darstellt.

Die genannten Buchpraktiken - insbesondere jene der Übersetzung, der Kompilation und der Auskoppelung - erlaubten es, rasch viele neue Bücher zu produzieren. Dies dadurch, dass aus bestehenden Büchern neue gemacht wurden. Dies verdeutlichte das Kapitel 5, das sich mit dem Wachsen des Weckerschen Textkorpus befasste. Es zeigte sich, dass insbesondere das Übersetzen, Kompilieren und das Auskoppeln geeignete Praktiken waren, um schnell und häufig zu publizieren. Wecker und seine Kollaborierenden verwendeten all diese Praktiken. Das Auskoppeln einzelner Kapitel zu einem neuen Buch erscheint dabei als die

924 Wecker, Johann Jacob: Brief an Theodor Zwinger. UB Basel, Frey-Gryn Mscr II 4:Nr. 328. 
am wenigsten offensichtliche Praktik, da die neuen Bücher andere Titel tragen trotz zum Teil gleicher Inhalte.

Auch nach Weckers Tod wurde auf die gleiche Art und Weise weiter produziert; seine Position im Kompilationsnetzwerk wurde von einer anderen Person eingenommen. In einem Falle war dies Anna Wecker, bei den anderen Veröffentlichungen wissen wir es nicht. Die eben genannten Praktiken wurden folglich nicht nur von Wecker und seiner Mitwirkenden ausgeübt - auch seine Nachfolgerinnen und Nachfolger, unter anderem Alexander Read, der 1660 die Eighteen Books of the Secrets herausgab, führten entsprechende Praktiken aus. ${ }^{925}$ Dies gilt zumindest für das Übersetzen und Kompilieren. Inwiefern sie auch auskoppelten, kann Gegenstand zukünftiger Untersuchungen sein.

Demnach wurde nicht nur bei den Weckerschen Kompilationen, sondern auch später Text durch Kompilationsnetzwerke produziert. Folglich waren sie und nicht etwa Wecker oder Alexander Read allein - Autoren ihrer Publikationen. Durch das Konzept des Kompilationsnetzwerks wird zudem die Frage der Autorschaft bei Kompilationen beantwortet: nicht ein Autor oder eine Autorin respektive ein Kompilator oder eine Kompilatorin standen hinter einem solchen Text, sondern ein Kompilationsnetzwerk. Damit wurde für den Kontext der Kompilationen die traditionelle Autorschaftsvorstellung hinterfragt, die sich etwa bei Albrecht Burckhardt findet, der bei Wecker von einem „unselbständige[n] Schriftsteller“ spricht. ${ }^{926}$ Das Kompilationsnetzwerk zeigt auf, dass das traditionelle Verständnis vom Autor als einzigartiger und insbesondere einzelner Kreator von Neuem hier nicht greift. ${ }^{927}$ Dies verdeutlicht insbesondere das zweite Kapitel. Darin konnte festgestellt werden, dass bisherige Modelle von Autorschaft der Komplexität der Autorschaft von Kompilationen nicht gerecht werden. Dies deshalb, weil für diese Texte nicht eine Autorin oder ein Autor verantwortlich war, sondern ein Geflecht unterschiedlicher Akteurinnen, Akteuren und Elementen, die relational miteinander interagierten, um eine Kompilation herzustellen. Demzufolge sollte nicht von einem „unselbständige[n] Schriftsteller“ gesprochen werden, sondern von einem Kompilationsnetzwerk, das Kompilationen produzierte. ${ }^{928}$ Das Konzept des Kompilationsnetzwerks erlaubt es folglich, die Komplexität der Autorschaft von solchen Texten aufzuzeigen und traditionelle Autorschaftsvorstellungen im Kontext von Kompilationen zu hinterfragen.

Dies ist insbesondere deshalb relevant, weil bei Publikationen wie den Books of Secrets unterschiedlichste Wissensbestände von verschiedenen „authores“

925 Wecker, Read: Eighteen Books of the Secrets of Art \& Nature, 1660.

926 Burckhardt: Geschichte der medizinischen Fakultät zu Basel 1460-1900, 1917. Zitat S. 55.

927 Siehe Kapitel 1, Einleitung.

928 Burckhardt: Geschichte der medizinischen Fakultät zu Basel 1460-1900, 1917, S. 55. 
zusammenkommen, die nicht von einer Person allein versammelt wurden, sondern von Kompilationsnetzwerken. Die Varietät von Wissensbestandteilen ist insbesondere bei der De Secretis libri XVII aus dem Jahr 1582 hoch, bei der das Weckersche Kompilationsnetzwerk vom reinen Übersetzen dazu überging, aus unterschiedlichsten Büchern zu kompilieren. Dadurch unterscheidet sich dieses Buch auch von anderen Books of Secrets der Zeit. In diesem Buch findet sich Wissen über Gott und die Engel, über Winde sowie darüber, wie eine Kerze unter Wasser brennen kann. ${ }^{929}$ Ihm ist ein universalistischer Anspruch inhärent, wie es charakteristisch für Enzyklopädien ist, weshalb hier auch von einer „Enzyklopädie der Geheimnisse“ gesprochen werden kann. ${ }^{930}$ Der Begriff der Enzyklopädie wurde im 16. Jahrhundert jedoch kaum verwendet, weshalb er wohl auch im De Secretis libri XVII nicht zu finden ist. ${ }^{931}$ Erst im 18. Jahrhundert wird vermehrt von Enzyklopädien gesprochen. ${ }^{932}$ Dieser Terminus wurde denn auch für die auf Englisch übersetzte und nochmals erweiterte Ausgabe aus dem Jahr 1660 verwendet, welche die Bezeichnung „Encyclipædie of Arts and Sciences“ enthält. ${ }^{933}$

Gemeinsam ist enzyklopädischen Publikationen der Anspruch auf ein systematisch geordnetes Wissen sowie auf eine Wissenstotalität. ${ }^{934}$ Rudolf Schenda verstand unter einer Enzyklopädie denn auch eine „geordnete Darstellung eines jeweils für wichtig erachteten und für einen größeren Kreis von Wissbegierigen

929 Ebd.

930 Wecker: De Secretis libri XVII, 1582. Der Begriff der Enzyklopädie ist kein, wie man es auf den ersten Blick vermuten könnte, antiker und tritt erstmals im 15. Jahrhundert auf, Bezug nehmend auf den orbis disciplinarium. Vogelsang: Zum Begriff ,Enzyklopädie‘, 2004, S. 19f. Dennoch sind auch vorher enzyklopädische Publikationen bekannt, dies seit der Antike. Meier: Einführung, 2002, S. 11, 16f.; Vogelsang: Zum Begriff ,Enzyklopädie‘, 2004, S. 19. Im 16. Jahrhundert wurden enzyklopädische Wissenssammlungen mit Titeln wie Summa, Collecteana, Compendium, Florilegium, Methodus, Theatrum oder Thesaurum versehen. Ebd., S. 22; Meier: Einführung, 2002, S. 13; Friedrich, Udo: Grenzen des Ordo im enzyklopädischen Schrifttum des 16. Jahrhunderts, in: Meier, Christel (Hg.): Die Enzyklopädie im Wandel vom Hochmittelalter bis zur frühen Neuzeit. Akten des Kolloquiums des Projekts D im Sonderforschungsbereich 231 (29.11.-1.12.1996) (= Münstersche Mittelalter-Schriften). München: Wilhelm Fink, 2002, S. 391-408, hier S. 392. Zur Entwicklung des Begriffs „Enzyklopädie“ siehe u. a. Vogelsang: Zum Begriff ,Enzyklopädie‘, 2004 und Dierse: Enzyklopädie, 1977.

931 Wecker: De Secretis libri XVII, 1582.

932 Schneider, Ulrich Johannes (Hg.): Die Erfindung des allgemeinen Wissens. Enzyklopädisches Schreiben im Zeitalter der Aufklärung. Berlin: De Gruyter; Akademie-Verl., 2013, S. 16.

933 Wecker, Read: Eighteen Books of the Secrets of Art \& Nature, 1660.

934 Friedrich: Grenzen des Ordo im enzyklopädischen Schrifttum des 16. Jahrhunderts, 2002, S. 392. 
brauchbaren Gesamtwissens.“935 Auch das „Kardinalproblem“ und gleichzeitig die „Hauptleistung“ der Enzyklopädie verbindet laut Christel Meier-Staubach die unterschiedlichen Arten derselben: Die Auswahl und die Ordnung des Wissens. ${ }^{936}$ Die Ordnung des Wissens konnte dabei auf unterschiedlichste Arten erfolgen, unter anderem auch durch ramistische Tabellen, wie sie von Theodor Zwinger, aber auch von Johann Jacob Wecker und seinen Kollaborierenden verwendet wurden. ${ }^{937}$

Auf diese Weise geordnet wurden auch die Wissensbestände der De Secretis libri XVII, die unterschiedlichen Wissenstraditionen entstammten: dazu gehören beispielsweise Anleitungen, die Galen und solche, die Paracelsus zugeschrieben werden. ${ }^{938}$ Auch sind sowohl „hermetische“ als auch „orthodoxe“ Bestände enthalten. Damit meine ich etwa Wissensbestände von Hermes Trismegistos und Albertus Magnus, oder aber von Aristoteles oder eben Galen. Das Zusammennehmen all dieser Bestände zeigt, dass diese Trennung zwischen „hermetisch“ und „orthodox“ für die Frühe Neuzeit nicht zwingend ist, was die Weckerschen Publikationen deutlich machen. In den De Secretis libri XVII ab 1582 finden sich neben zeitgenössischem Textelementen auch antike, wobei das Wissen für die englische Ausgabe Eighteen Books of the Secrets noch einmal aktualisiert wurde. ${ }^{939}$ Diese Bücher enthalten Informationen, die über 129 „authores“ zugeschrieben wurden. ${ }^{940}$ Genauer gesagt wurde das Wissen anderen Büchern entnommen und nicht etwa der Beobachtung der Natur, der Durchführung von Experimenten oder anderen Techniken der Wissensgenerierung. Bereits bestehende Bücher bildeten die Grundlage der Textproduktion der De Secretis libri $X V I I$, wie die vorliegende Studie zeigen konnte.

Das Konzept des Kompilationsnetzwerks wurde anhand der Weckerschen Buchproduktion ausgearbeitet, ist aber auch auf andere zeitgenössische Kompilationsproduktionen übertragbar. So etwa auf enzyklopädisch ausgerichtete $\mathrm{Pu}$ -

935 Schenda, Rudolf: Hand-Wissen. Zur Vorgeschichte der grossen Enzyklopädien, in: Zürich, Universität (Hg.): Populäre Enzyklopädien. Von der Auswahl, Ordnung und Vermittlung des Wissens. Zürich: Chronos Verlag, 2002, S. 15-34, hier S. 21. Nach Ulrich Dierse bezeichnet der Begriff der Enzyklopädie „die systematische Erfassung, Anordnung und Lehre des Wissens; er steht ursprünglich für die Klassifikation der Wissenschaften und deren theoretische Grundlegung.“ Ders.: Enzyklopädie, 1977, S. 339.Vgl. Conrad, Ruth: Lexikonpolitik. Die erste Auflage der RGG im Horizont protestantischer Lexikographie. Berlin: De Gruyter, 2006, S. 32.

936 Meier: Einführung, 2002, S. 17.

937 Siehe dazu Kapitel 4.4, Wissen ordnen.

938 Wecker: De Secretis libri XVII, 1582, passim.

939 Ebd.; Wecker, Read: Eighteen Books of the Secrets of Art \& Nature, 1660.

940 Wecker: De Secretis libri XVII, 1582. 
blikationen wie Spiegel, Theatri, Bibliothecae oder Pandectae. ${ }^{941}$ Dahinter stand nach Ann Blair eine gewisse „info-lust“, die auch bei den Weckerschen Kompilationsnetzwerken ausgemacht werden kann. ${ }^{942}$ Um dieser nachzugehen und an das gewünschte Wissen zu gelangen, war eine Kollaboration notwendig, weshalb hinter all diesen Texten Kompilationsnetzwerke angenommen werden können. Dies gilt jedoch nicht nur für enzyklopädisch orientierte Publikationen: Hinter vielen frühneuzeitlichen Texten standen kompilatorische Textproduktionspraktiken. Dazu zählen unter anderem Tierbücher, Kosmographien, Herbarien, Florilegiensammlungen, Texte der Buntschriftstellerei, Flugblätter, Nachrichtensammlungen und Briefsummarien. ${ }^{943}$ Auch literarische Texte wie Johannes Fischarts Affentheurlich Naupengeheurliche Geschichtklitterung oder der 1509 publizierte juristische Laÿen Spiegel von rechtmässigen ordnungen in Burgerlichen vnd peinlichen regimenten weisen kompilatorische Elemente auf. ${ }^{944}$ Dabei liesse sich fragen, inwiefern sich Kompilationsnetzwerke von literarischen von solchen nicht literarischer Texte unterscheiden - eine Frage, die der zukünftigen Forschung übertragen wird.

Die vorliegende Arbeit untersuchte die Textproduktionspraktiken und die Autorschaft frühneuzeitlicher Kompilationen, wobei der Fokus auf Books of Secrets lag. Doch wie entwickelten sich diese weiter? Am Beispiel Weckers wie auch anhand der Forschungsliteratur zeigt sich, dass Books of Secrets ein frühneu-

941 Siehe u.a. Schock: Polyhistorismus und Buntschriftstellerei, 2012; Ders.: Wissensliteratur und ,Buntschriftstellerei' in der Frühen Neuzeit, 2012; Zedelmaier: Navigieren im Textuniversum, 2008.

942 Blair: Too Much to Know, 2010, S. 6; Dies.: Reading Strategies for Coping With Information Overload ca. 1550 -1700, 2003, S. 11.

943 Siehe u. a. Zedelmaier: Navigieren im Textuniversum, 2008, insbes. S. 114; Schierbaum: Enzyklopädistik 1550 -1650, 2009; Stammen, Weber, Wolfgang E. J: Wissenssicherung, Wissensordnung und Wissensverarbeitung, 2004; Schock: Polyhistorismus und Buntschriftstellerei, 2012; Ders.: Wissensliteratur und ,Buntschriftstellerei' in der Frühen Neuzeit, 2012; Harms, Schilling: Das illustrierte Flugblatt der frühen Neuzeit, 2008; Schilling: Flugblatt und Drama in der Frühen Neuzeit, 2008, u. a. S. 243f.; Zwierlein: Fuggerzeitungen als Ergebnis von italienisch-deutschem Kulturtransfer 1552-1570, 2010, S. 177. Siehe dazu Zweifel: Ein Blick hinter die Produktion von Kompilationen im 16. Jahrhundert am Beispiel Johann Jacob Weckers, 2018.

944 Fischart, Johannes: Affenteurliche vnd Vngeheurliche Geschichtschrift Vom Leben rhaten vnd Thaten der [...] Helden vnd Herrn Grandgusier Gargantoa vnd Pantagruel Königen inn Vtopien vnd Ninenreich. Etwan von M.Francisco Rabelais Französisch entworfen: Nun aber [...] auf den Teutschen Meridian visirt [...] durch Huldrich Elloposcleron Reznem. Straßburg: Bernhard Jobin, 1575; Tengler, Ulrich; Brant, Sebastian; Locher, Jacobus: Laÿen Spiegel: Von rechtmässigen ordnungen in Burgerlichen vnd peinlichen regimenten [...]. Augspurg: Otmar, 1509. 
zeitliches Phänomen waren. ${ }^{945}$ Teile dieser Bücher sind in andere Sammlungen aufgegangen, d. h., dass aus ihnen weiter kompiliert wurde. Ein Beispiel dafür aus den Weckerschen Secreti ist die Anleitung zum Bau von Drachen, die sich in Daniel Schwenters Deliciæ physico-mathematicæ (1651) mit Bezugnahme auf Wecker findet. ${ }^{946} 1799$ wurde diese Anleitung von Friedrich Murhard in die Geschichte der Physik übernommen. ${ }^{947}$ Auch in Johann Jacob Scheuchzers NaturHistorie des Schweitzerlandes (1716) ist eine Spur Weckers $\mathrm{zu}$ entdecken: Scheuchzer hat Wissen über ,goldhaltende Bäder“ in sein Buch integriert. ${ }^{948}$ Die letzten beiden Publikationen stammen aus dem 18. Jahrhundert; im 19. Jahrhundert verblassen die Spuren der Secreti. Mit ihnen verschwindet auch eines ihrer wichtigen Merkmale: das Werben mit der Vielfalt der Inhalte sowie der Autoritäten, denen das Wissen entnommen worden sei.

Dies gilt zumindest für Enzyklopädien nach den „Enzyklopädien der Geheimnisse“, für solche des 18. Jahrhunderts - man denke etwa an etwa an Zedlers Großes vollständiges Universal-Lexicon aus dem Jahr 1732, an die Encyclopédie, ou Dictionnaire raisonné des sciences, des arts et des métiers von Denis Diderot und Jean le Rond D'Alembert sowie an die Encyclopaedia Britannica, die in der zweiten Hälfte des 18. Jahrhunderts erschienen sind. ${ }^{949}$ Trotz gleichbleibendem univer-

945 Siehe Martins: Les livres de secrets imprimés et traduits en Europe, 2015, S. 146; Gruman Martins: Os livros de segredos italianos e o desenvolvimento da ciência moderna, 2014, S. 221f. Das letzte Piemontesesche Book of Secret wurde laut William Eamon 1780 veröffentlicht. Ders.: Science and the Secrets of Nature, 1996, S. 357. Auch die Forschungsliteratur zu Books of Secrets geht nicht über das 18. Jahrhundert hinaus. Siehe u. a. ebd.; Kavey: Books of Secrets, 2007; Bela: The Authorship of the „Secrets of Alexis of Piedmont“, 2016; Gulizia: Ruscelli’s Book of Secrets in Context, 2014.

946 Schwenter, Daniel: Deliciæ physico-mathematicæ oder Mathemat. Und philosophische Erquickstunden: darinnen sechshundert dreÿ und sechzig, schone, liebliche und annehmliche Kunststücklein, Auffgaben und Fragen, auss der Rechenkunst, Landtmessen, Perspectiv, Naturkündigung, und andern Wissenschafften genomen, begriffen seindt. Nürnberg: In Verlegung Jeremiæ Dümlers, 1651, S. 472-474.

947 Murhard, Friedrich W.A.: Geschichte der Physik seit dem Wiederaufleben der Wissenschaften bis an das Ende des achtzehnten Jahrhunderts. Die Geschichte der Montgolfieren und Barometrie enthaltend (= Bd. 1, Nr. 1). Göttingen: Rosenbusch, 1799, S. 33-36.

948 Scheuchzer, Johann Jacob: Helvetiæ historia naturalis oder Natur-Historie des Schweitzerlandes. Zürich: In der Bodmerischen Truckerey, 1716, S. 152.

949 Zedler, Johann Heinrich: Grosses vollständiges Universal-Lexicon aller Wissenschafften und Künste, Welche bißhero durch menschlichen Verstand und Witz erfunden und verbessert worden [...]. Halle; Leipzig: Zedler, 1732-1754; Diderot; Le Rond d'Alembert: Encyclopédie, ou Dictionnaire raisonné des sciences, 1780; Society of Gentlemen in Scotland (Hg.): Encyclopædia Britannica, or, A dictionary of arts and sciences, compiled upon a new plan [...]. Edinburgh: A. Bell and C. Macfarquhar, 1768-1771. 
salistischem Anspruch wird hier nicht mehr die Diversität der „authores“, denen Wissen entnommen wurde, betont. Diese wurde überschrieben, um die Autorität und Originalität des Autors stark zu machen. ${ }^{950}$ Damit einher ging die Repräsentation der Autoren der Enzyklopädien als „Genies“, die hinter diesen Texten standen. ${ }^{951}$ Diese Repräsentation kann man mit der in dieser Zeit stärker werdenden, genieästhetischen Idee des Autors in Verbindung bringen: Auch Enzyklopädien sollen nun, wie etwa im Fall der Encyclopédie, von „Genies“ erschaffen worden sein.

Nicht verschwunden hingegen ist die Kompilation als Praktik der Textproduktion. Auch die Enzyklopädien des 18. Jahrhunderts enthalten schon vorher existierende Wissensbestände, die etwa übersetzt und in die neuen Bücher eingefügt wurden. ${ }^{952}$ Auch waren mehrere Akteurinnen und Akteure sowie Elemente in die Produktion dieser Texte involviert, doch wurde dies viel weniger sichtbar gemacht. Verändert hat sich zudem die textuelle Repräsentation: Waren bei Wecker die entnommenen Textstellen zumindest bis zu einem gewissen Grad zuweisbar, so wurde dieser Link zu den Büchern als Ursprungsmaterialien weggelassen. Dadurch entstand eine Lücke zwischen Praktik und Repräsentation: Während die Buchpraktik der Kompilation noch immer Bestand hatte, wurde sie aufgrund der Repräsentation eines Autors - oder eines anonymen Autors - als Urheber einer Enzyklopädie sowie durch das Weglassen der Textverweise verschleiert. ${ }^{953}$

Doch auch Verweise auf bestehende Texte sind nicht abhanden gekommen. Sie finden sich unter anderem in Form von Fussnoten im geisteswissenschaftlichen Buch wieder; solche wurden schon im 18. Jahrhundert verwendet. ${ }^{954}$ Fussnoten rekurrieren immer auf einen bereits bestehenden Text, der vorhanden sein musste, damit diesem Wissen entnommen werden konnte. Damit liegt eine ähnliche Voraussetzung vor wie bei frühneuzeitlichen Kompilationen, bei denen ebenfalls ein Buch vorliegen musste, um neuen Text herstellen zu können. Der Unterschied liegt vor allem in der Materialität, der Beschaffung und dem Umgang mit dem bereits bestehenden Text: Waren die frühneuzeitlichen Bücher als Codizes oder Abschriften derselben $\mathrm{zu}$ beschaffen, so sind heute auch digitale Bücher Teil der Grundlage von neuen Büchern. Zudem müssen für die Beschaffung

950 Hirschi: Compiler into Genius, 2013, S. 150.

951 Ebd.

952 Siehe dazu ebd., S. 147.

953 Vgl. ebd., S. 164.

954 Beispielsweise in: Gibbon, Edward: The History of the Decline and Fall of the Roman Empire (= Bd. 8). London: W. Strahan; T. Cadell [...] and W. Davies, 1797. Vgl. Grafton: The Footnote, 1997, S. 1 . 
weder Botinnen noch Boten agieren, noch ist eine ausführliche Korrespondenz nötig, um sich über mögliche Bücher als Basis des neuen Buches informieren zu können. Des Weiteren wurden Textelemente direkt, manchmal gekürzt und neu geordnet, aber ohne diese umzuschreiben, in das neue Buch übertragen. Folglich unterscheidet sich der Grad der Verflechtung der einzelnen Textteile: Sind bei frühneuzeitlichen Kompilationen die Elemente aneinandergereiht, so werden sie im geisteswissenschaftlichen Schrifttum miteinander verwoben. Dies verdeutlicht etwa eine Fussnote mit vier verschiedenen Referenzen: all diese Bezugnahmen werden zu einer einzelnen Aussage zusammengenommen. Dabei geht es meist nicht mehr um eine direkte Übernahme von Text, sondern von einer Idee, die von all den vier Autorinnen und/oder Autoren, die in der Fussnote genannt werden, beschrieben wurde. Hinter diesen Autorinnen und/oder Autoren steht meist wiederum ein Geflecht von verschiedenen Akteurinnen, Akteuren und Elementen, so dass das Gesamtgeflecht der geisteswissenschaftlichen Textproduktion stark verwoben und sehr dicht sein kann. Zudem wird in geisteswissenschaftlichen Schriften mehr Text neu verfasst, die direkte Übernahme bleibt den Zitaten vorbehalten. Bei Nichtberücksichtigung dieser Praktik droht ein Plagiatsvorwurf.

Wie bei frühneuzeitlichen Kompilationen ist auch bei geisteswissenschaftlichen Veröffentlichungen das soziale Netzwerk bedeutsam: Personen diskutieren Textentwürfe, geben neue Ideen und helfen auch einmal, einen digitalen Text zu beschaffen, zu dem man selbst keinen Zugang hat. Reviewer von Büchern können einen Aufsatz oder ein Buch ebenfalls beeinflussen, dies, indem sie viele Kritikpunkte rückmelden, ohne deren Berücksichtigung ein Text nicht publiziert wird. Demnach weist auch ein geisteswissenschaftliches Buch Aspekte eines Kompilationsnetzwerks auf. Es unterscheidet sich jedoch in der stärkeren Verflochtenheit des Basistextes, in der grösseren Bedeutung des Neuschreibens von Text und in der ebenfalls bestehenden teilweisen Lücke zwischen Praktik und Repräsentation von Textproduktion und Autorschaft von der frühneuzeitlichen Kompilation. Teilweise deshalb, weil einige Autorinnen und Autoren viele Personen angeben, die an der Textproduktion beteiligt waren und auch, weil Bezüge zu den Basistexten anhand von Fussnoten gesetzt werden. Dadurch unterscheiden sie sich strukturell auch von den Enzyklopädien des 18. Jahrhunderts.

Enzyklopädien werden auch in der Moderne verfasst. Auch diese weisen kompilatorische Elemente auf, wie beispielsweise bei wikipedia.org. ${ }^{955}$ Darin findet sich Text, der an jenen des geisteswissenschaftlichen Buchs erinnert, der durch Fussnoten auf bereits vorher bestehenden Text verweist. Damit wird das kompilatorische Element des geisteswissenschaftlichen Buchs aufgegriffen. Im

955 Wikipedia. Online unter: www.wikipedia.org [23.02.2021]. 
Unterschied zu letzterem steht hier jedoch ein kollaboratives Erarbeiten von Text im Vordergrund; die Autorschaft des einzelnen Artikels ist nicht bedeutsam. Dennoch wird neuer Text geschrieben, der bestehende also stark modifiziert. Dabei lässt sich der Textproduktionsverlauf, im Gegensatz zu den meisten anderen Texten, nachverfolgen. Die häufig vielen Personen, die in die Textproduktion involviert waren, werden sichtbar, weshalb das Geflecht der Textproduktion deutlicher hervortritt als etwa beim geisteswissenschaftlichen Buch. Ein wichtiger Faktor ist hingegen nicht involviert, der sowohl beim geisteswissenschaftlichen Buch als auch bei Books of Secrets äusserst bedeutsam war respektive ist: die Frankfurter Buchmesse. Aufgrund der anderen Materialität des Textes verändern sich folglich die an der Buchproduktion beteiligten Elemente. Auch das Buch in seiner Materialität - bei frühneuzeitlichen Books of Secrets als Codex - ist nicht mehr gleich relevant und damit auch dessen Beschaffung und die daran beteiligten Personen. Demnach ändern sich die Akteurinnen und Akteure sowie Elemente und beeinflussenden Faktoren je nach kompilatorischer Textproduktion; der geflechtartige Charakter bleibt jedoch bestehen. Dieser, wie auch die Praktik, mit bestehendem Textmaterial zu arbeiten, findet sich in vielen, auch modernen, Textgattungen. Aus diesem Grund ist es wichtig, über Textproduktionsgeflechte anderer Genres und Zeiten nachzudenken, auch, um gängige Autorschafts- oder Nichtautorschaftsvorstellungen zu hinterfragen. Das frühneuzeitliche Kompilationsnetzwerk bildet die Grundlage dafür.

Kompilationsnetzwerke helfen nicht nur, ein neues Verständnis von Autorschaft zu gewinnen. Sie waren bei der Herstellung von Books of Secrets in der Frühen Neuzeit die grundlegende Voraussetzung für deren Erfolg. Sie ermöglichten es, rasch viel Wissen zu versammeln und dieses immer wieder neu $\mathrm{zu}$ publizieren. Die wichtigste Buchpraktik dabei war jene der Kompilation, ergänzt durch jene der Übersetzung und des Auskoppelns von Text. Diese Praktiken erlaubten es, viele unterschiedliche Wissensbestände zu versammeln und das Buch „[...] so groß [zu machen, SZ], als mir müglich gwesen [...]. “956 Die gesammelten Wissensbestände wurden immer wieder neu und in veränderten Ausgaben herausgegeben, so dass eine Multiplikation von Büchern erfolgte, die mit einer Multiplikation von Wissen einherging. Dahinter stand die Kompilation als wesentliche Buchproduktionspraktik: Sie bildete die Grundlage der Erfolgsstory der Books of Secrets.

956 Wecker, Johann Jacob: Brief an Theodor Zwinger. UB Basel, Frey-Gryn Mscr II 4:Nr. 326. Zitat siehe auch S. 66 sowie 193. 